\title{
Mutation of PD-1 immune receptor tyrosine-based switch motif (ITSM) enhances the antitumor activity of cytotoxic T cells
}

\author{
Tongbing $\mathrm{Qi}^{1 \#}$, Juan Fu ${ }^{2 \#}$, Wen Zhang ${ }^{3 \#}$, Weitong Cui ${ }^{1}$, Xiaosong Xu ${ }^{2}$, Jianmei Yue ${ }^{4}$, Qinglu Wang ${ }^{1}$, \\ Xuewen $\operatorname{Tian}^{5}$ \\ ${ }^{1}$ Key Laboratory of Biomedical Engineering \& Technology of Shandong High School, Qilu Medical University, Zibo, China; ${ }^{2}$ Shandong Bio-focus \\ Gene Science and Technology Ltd., Zibo, China; ${ }^{3}$ Clinical laboratory of Zibo Central Hospital, Zibo, China; ${ }^{4}$ Department of Endocrinology, the \\ First Hospital of Zibo City, Qilu Medical University, Zibo, China; ${ }^{5}$ College of Sports and Health, Shandong Sport University, Jinan, China \\ Contributions: (I) Conception and design: Q Wang; (II) Administrative support: X Tian; (III) Provision of study materials or patients: W Cui; (IV) \\ Collection and assembly of data: T Qi, X Xu; (V) Data analysis and interpretation: J Yue, W Zhang; (VI) Manuscript writing: All authors; (VII) Final \\ approval of manuscript: All authors. \\ \#These authors contributed equally to this work. \\ Correspondence to: Dr. Qinglu Wang. Key Laboratory of Biomedical Engineering and Technology of Shandong High School, Qilu Medical University, \\ 2018 Jiangmeng Road, Economic Development Zone, Zibo 255300, China. Email: wq1_zcq@126.com; Xuewen Tian. Department of Sports and \\ Health, Shandong Sport University, 10600 Century Avenue, Licheng, Jinan 250102, China. Email: xuewentian1978@163.com.
}

Background: Programmed cell death protein 1 (PD-1), as an immune checkpoint cell membrane receptor, negatively regulates $\mathrm{T}$ cell activation via its immune receptor, the tyrosine-based switch motif (ITSM). The purpose of this research was to evaluate the antitumor activity T cells with the ITSM mutation of PD-1 on non-small cell lung cancer (NSCLC) in vitro and in vivo.

Methods: In this study, the tyrosine of ITSM in cytotoxic T cells was mutated using the adenine base editor (ABE)-xCas9 system to evaluate its effect on the antitumor activity of T cells against NSCLC.

Results: Results showed that the PD-1-deficient T cells enhanced the death of the cocultured NSCLC cells compared with the normal T cells and saline solution. PD-1-deficient $\mathrm{T}$ cells also changed the interleukin 2(IL-2), interferon $\gamma$ (IFN- $\gamma$ ), tumor necrosis factor $\alpha$ (TNF- $\alpha$ ), and granulocyte-macrophage colony-stimulating factor (GM-CSF) secretion of T cells compared with those of the normal T cells. The effectiveness of ITSM mutation in enhancing the antitumor activity of PD-1-deficient T cells was verified in vivo by using a mouse xenograft model. The xenografted mice treated with PD-1-deficient $\mathrm{T}$ cells demonstrated repressed tumor growth of the NSCLC cells compared with those treated with normal T cells and saline solution.

Conclusions: The mutation of ITSM in cytotoxic T cell via the ABE-xCas9 system can significantly enhance the antitumor activity of T cells.

Keywords: Antitumor immunity; immune receptor tyrosine-based switch motif (ITSM); adenine base editorxCas9 system (ABE-xCas9 system); tyrosine mutation; non-small cell lung cancer cells

Submitted May 18, 2020. Accepted for publication Oct 09, 2020.

doi: $10.21037 /$ tcr-20-2118

View this article at: http://dx.doi.org/10.21037/tcr-20-2118

\section{Introduction}

Programmed cell death protein 1 (PD-1) and its ligand PDL1/L2 have recently attracted attention from researchers. Monoclonal antibodies targeting PD-1 or PD-L1/L2, such as nivolumab, pembrolizumab, and atezolizumab, have been developed and used in clinical trials (1). Furthermore, tumor immunotherapy based on the PD-1/PD-L1/L2 immune checkpoint blockade has demonstrated considerable efficacy 
in the clinical trials of various cancers $(2,3)$. PD-1 and PD-L1 are membrane protein receptors with canonical immunoglobulin-like extracellular domains responsible for interaction and signal transduction to intracellular domains.

The T cell of PD-1 knockout results in the significant reduction in PD-1 expression but does not affect the viability of primary human $\mathrm{T}$ cells during the prolonged in vitro culture. In addition, this kind of cell is characterized by upregulated interferon $\gamma(\mathrm{IFN}-\gamma)$ production and enhanced cytotoxicity (4). Further studies have shown that PD-1 knockout $\mathrm{T}$ cells exert an impressive antitumor effect in a xenograft mouse model (5-7).

Chimeric antigen receptor (CAR) $\mathrm{T}$ cell therapies have demonstrated immense therapeutic potential, but its application in solid tumor is restricted due to the tumor microenvironment. Immune checkpoint is one of the important factors in the formation of tumor microenvironment. PD-1-defective CAR T cell therapy has significantly enhanced the $\mathrm{T}$ cell antitumor activator $(8,9)$. In a recent report from our group, multiplex genome editing is achieved using the CRISPR/Cas9 system to simultaneously disrupt PD-1 and T cell receptor (TCR) alpha and beta chains in the NY-ESO1 TCR-engineered T cells (10).

PD-1 consists of a single N-terminal immunoglobulin variable region (IGV)-like domain, a stem region that separates the IGV domain from the plasma membrane and contains about 20 amino acids, a transmembrane region, and a cytoplasmic tail region that contains a tyrosine signal motif [immune receptor tyrosine-based inhibition motif (ITIM)] $(11,12)$. The function of ITIM is still not clear. When the tyrosine inhibitory motif of the immune receptor changes to phenylalanine (y223f), the inhibition of PD-1 is retained, but when the immune receptor tyrosine-based switch motif (ITSM) of the immune receptor changes (y248f), this inhibition is lost (13).

In recent years, CRISPR/Cas9 has become the primary tool of gene editing due to its high efficiency. CRISPR/ Cas9 has been widely used in the diagnosis and treatment of diseases $(14,15)$. The CRISPR/Cas9 gene editing system is simple and efficient, but its site-specific modification depends on the inefficient homologous recombination mechanism. Therefore, the ability of precise gene editing needs to be improved (16). The base editor can edit bases directly without cutting the DNA and the RNA sequences and has an editing efficiency of over $50 \%$, which is higher than CRISPR, and almost no adverse side effects. The single base-editing technology provides an important tool for the research and application of gene editing technology (17-20). In the prior art, the T-lymphocyte preparation used for tumor treatment has serious side effects, complex preparation process, long cycle, and high cost. The present study provides a preparation method for PD-1-deficient T-lymphocyte, which simplifies the production process of cells, improves the speed of preparation, reduces the cost, and produces minimal side effects of T-lymphocyte preparation.

We present the following article in accordance with the ARRIVE reporting checklist (available at http://dx.doi. org/10.21037/tcr-20-2118).

\section{Methods}

\section{Animals model}

Thirty A549 (non-small cell lung cancer cell) xenograft mouse models (NOD-SCID) were provided by JRDUN Biotechnology Co. Ltd., Shanghai (certificate number: scxk). The mice were raised at room temperature (approximately $20{ }^{\circ} \mathrm{C}$ ) and $60 \%$ relative humidity with a 12 -h light/dark cycle. The mice were allowed to adapt to the experimental environment for one week. Afterward, blood was taken from the canthus of mice. The experiments were conducted in accordance with the guidelines on the care and use of experimental animals issued by the Ministry of Health of the People's Republic of China and the guidelines on the care and use of animals in the IJSM ethical standard documents, and in accordance with the project license (No. 20200001) granted by the Animal Experiment Committee of Qilu Medical University.

\section{Plasmid expression vectors}

The full PD-1 gene sequence (ef064716.1) was obtained from NCBI, and the corresponding gene sequence of the ITSM region was identified. The gRNA target sequence was designed on the basis of the CRISPR-DO website, and a suitable target sequence ( $20 \mathrm{bp}$ length) was selected in accordance with the set parameters. Then, the guide RNA (gRNA) target sequence, adenine base editor (ABE), xCas9,

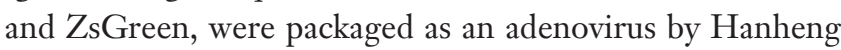
Biotechnology (Shanghai) Co., Ltd.

\section{Isolation and activation of T lymphocytes}

PBS buffer $(2 \mathrm{~mL} /$ well $)$ was placed in a 6 -well plate. 
Subsequently, the $\mathrm{CD}^{+}$antibody was added to obtain a final concentration of $10 \mu \mathrm{g} / \mathrm{mL}$. The mixture was incubated in a $\mathrm{CO}_{2}$ incubator at $37^{\circ} \mathrm{C}$ for $2 \mathrm{~h}$, and PBS was removed from the mixture. The plate was washed twice with fresh preheated PBS and washed once with serum-free RPMI1640 medium. Fresh blood $(1 \mathrm{~mL})$ was extracted from the mouse canthus and immediately added into a $1.5-\mathrm{mL}$ centrifuge tube containing $10 \mu \mathrm{L}$ heparin anticoagulant. Blood samples were immediately separated to maintain the activity of lymphocytes. PBS or $0.9 \% \mathrm{NaCl}(3 \mathrm{~mL})$ was added to dilute blood or plasma. The dilution of blood can reduce the aggregation of red blood cells and increase the harvest of lymphocytes. The lymphocyte separation solution $(4 \mathrm{~mL})$ was collected, added to the bottom of a centrifuge tube, and heated to room temperature. Diluted blood sample $(4 \mathrm{~mL})$ was collected using a glass Pasteur pipette and slowly spread along the wall of the pipette onto the lymphocyte separating solution. Moreover, the stability of the liquid interface was maintained. The horizontal rotor was used for centrifugation at 2,000 RPM or $700 \times \mathrm{g}$ and $20{ }^{\circ} \mathrm{C}$ for $20 \mathrm{~min}$. Blood stored for more than $2 \mathrm{~h}$ was centrifuged for $30 \mathrm{~min}$. After centrifugation, the bottom of the tube contained the red blood cells, the middle layer was the separating liquid, and the top layer contained the plasma. Between the plasma layer and the separation solution was a thin, dense white membrane containing monocytes (including lymphocytes and monocytes). A pipette was inserted directly into the monocyte layer, which was removed and placed into another tube. Hank's solution $(10 \mathrm{~mL})$ was added to dilute the separated lymphocytes. The cells were centrifuged at $250 \mathrm{RPM}$ for $10 \mathrm{~min}$, and the supernatant was discarded. The cells were washed twice to remove the platelets and the anticoagulants. Finally, the cells were counted through dilution with PBS 10 times and transferred to 6-well plates covered with $\mathrm{CD}^{+}$antibody.

\section{Flow cytometry and sequencing analysis}

After $48 \mathrm{~h}$ of adenovirus infection, the cells were washed twice with PBS, and then separated using $1 \mathrm{~mL}$ PBS buffer through flow cytometry. The GFP-positive $\mathrm{CD} 8^{+} \mathrm{T}$ cells were sorted for mutation site (y248f in ITSM) analysis. Genomic DNA was extracted by phenol-chloroform and alcohol precipitation. The targeted region of $\mathrm{PD}-1$ was PCR amplified (PD-1f: 5'-gacatttcaggaggaggagcattg-3'; PD-1r: 5'-catgtacctccccctgt-3') from genomic DNA using rTaq (Takara, DR001BM), and the products were purified using the PCR cleanup kit (Axygen, APPCR-50). The purified PCR product was sequenced by Shenggong Biotechnology (Shanghai) Co., Ltd. Target base-editing technology can offer $70-80 \%$ editing efficiency $(21,22)$. Thus, GFP-positive CD8 $8^{+} \mathrm{T}$ cells were used in the next experiments.

Apoptotic cell death was determined by flow cytometry analysis using annexin V-FITC and propidium iodide assay kit (BD, Pharmingen, San Diego, CA, USA). Control $\mathrm{T}$ and ITSM mutation $\mathrm{T}$ cells were collected, washed with cold PBS, suspended in $5 \mu \mathrm{L}$ of annexin $\mathrm{V}$ binding buffer, and stained with $5 \mu \mathrm{L}$ of propidium iodide. The cells were mixed gently, incubated in the dark for $20 \mathrm{~min}$, and washed. The samples were analysed with a FACS (Beckman Coulter, CA, USA).

\section{Analysis of the proliferation ability of ITSM mutation T cells}

Control $\mathrm{T}$ and ITSM mutation $\mathrm{T}$ cells $\left(1 \times 10^{3}\right.$ cells $)$ were cultured in RPM-1640 medium supplemented with interleukin (IL)-2 (300 U/mL) and half replaced by fresh complete medium containing IL-2 every $2-3$ days. The cell number was counted once every three days, and the growth curve was drawn in accordance with the cell expansion number.

\section{Cytotoxic test and cytokine release assay}

The $\mathrm{T}$ and the ITSM mutation $\mathrm{T}$ cells were cocultured with A549 cells in accordance with the effective target ratios of $1: 1,2: 1,4: 1$, and $8: 1$. The killing effect of the two groups on the target cells was tested $24 \mathrm{~h}$ later.

Simultaneous with detecting the killing effect of $T$ cells, the release of various cytokines was detected under the condition of coculture with target cells. The target cells $\left(1 \times 10^{4} /\right.$ well $)$ were placed in a 96 -well plate, and the required $\mathrm{T}$ cells or PD-1-deficient T cells $\left(2 \times 10^{4} /\right.$ well $)$ were added. Each $\mathrm{T}$ cell was divided into three kinds of multiple wells. The RPM-1640 medium $(200 \mu \mathrm{L})$ was added and cultured in an incubator maintained at $37^{\circ} \mathrm{C}$. After $24 \mathrm{~h}$, the liquid was removed in each well and centrifuged at $500 \times \mathrm{g}$ for $5 \mathrm{~min}$, and the supernatant was collected. The supernatant $(200 \mu \mathrm{L})$ was used to detect the cytokines in each reaction. The cytokine concentrations of IL-2, IFN- $\gamma$, tumor necrosis factor $\alpha(\mathrm{TNF}-\alpha)$, and granulocyte-macrophage colonystimulating factor (GM-CSF) were detected using the kit. 


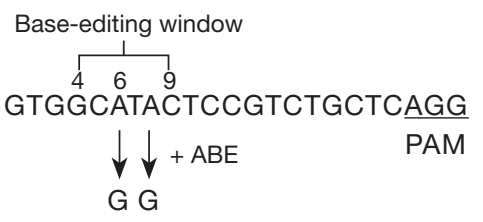

Figure 1 Sequence of target gRNA. After xCas9 and gRNA binding target sequence, adenine base editing (ABE) enables enzymatic conversion from A-T into G-C base pairs. gRNA, guide RNA.

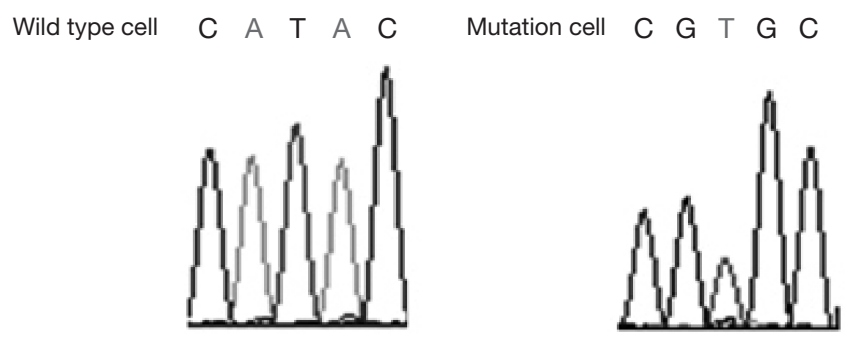

Figure 2 Sequence results of mutated site. Adenine base editing (ABE) converted A-T into G-C base pairs in mutation cell from sequence results of wild type cell line and mutation cell line.

\section{T cells killing ability assay}

One $\times 10^{5}$ A549 cells were incubated in a 6-well plate for $48 \mathrm{~h}$. Then $2 \times 10^{7} \mathrm{~T}$ or PD-1-deficient $\mathrm{T}$ cells were added into 6-well plate and co-incubated with A549 cells at an effector to target ratio $(\mathrm{E}: \mathrm{T})=1: 1,2: 1,4: 1$ and $8: 1$ for $48 \mathrm{~h}$, respectively. Next, T or PD-1-deficient T cells were removed and A549 cells were digested by trypsin for counting. Another group A549 cells served as a control for calculating A549 cells lysis (\%).

\section{Mouse xenograft assay}

The efficacy of PD-1-deficient $\mathrm{T}$ cells in repressing tumor growth was validated in vivo by using a human tumor xenograft model (A549 cell). Two of the three groups of mice were intravenously inoculated in the tail with $5 \times 10^{6} \mathrm{~T}$ and PD-1-deficient T cells. Another group (control group) was intravenously inoculated in the tail with saline solution. The mice were treated with either saline solution, $\mathrm{T}$ cells, or PD-1-deficient $\mathrm{T}$ cells four times to evaluate the repression of PD-1-deficient T cells on tumor growth. Simultaneously, the anal temperature of the mice in each group was measured for 7 consecutive days. The change in the body weight of mice was measured in four weeks.
The length and diameter of the tumor in mice were measured using the Vernier caliper, and tumor volume was calculated using the formula: $V=a \times B^{2} / 2$. $a$ is the longest diameter of tumor, and $B$ is the shortest diameter of tumor.

\section{Statistical analysis}

All data were analyzed using the SPSS13.0 statistical software. Results were expressed as means and standard deviation. For the comparison of any two groups, the $\mathrm{P}$ values were calculated using ANOVA with a post hoc test. $\mathrm{P}<0.05$ indicated a significant difference, and $\mathrm{P}<0.01$ indicated a highly significant difference.

\section{Results}

\section{Design and validation of sgRNA-targeting PD-1}

ABEs that mediate the conversion of $A^{*} T$ to $G^{*} C$ in genomic DNA had a specific window (4-6 bases) to edit the base in the corresponding sequence of gRNA. On the basis of the CRISPR-DO website and further gRNA sequence analysis, the appropriate gRNA sequence was obtained (Figure 1).

\section{Sequencing analysis of ITSM single base mutations in GFP-positive cells}

In the base-editing experiment, $44.7 \%$ of the cells had an effective point-to-point mutation from a to $g$ at two sites. Most (89.6\%) of the cells had an effective point-to-point mutation from a to $g$ at a single site (either single or two sites changed from a to $\mathrm{g}$ ), which resulted in the tyrosine modification of ITSM and the loss of PD-1 inhibition function (Figure 2). The sequencing results of 15 PCR products showed that only one did not develop point-topoint mutation. The base-editing technology of tyrosine in ITSM can offer a sufficient number of T-lymphocyte groups that can be disinhibited by the PD-1 immune checkpoint. This kind of cell group can block the signal pathway of PD-1-inducing $T$ cell apoptosis and retain the other functions of $\mathrm{PD}-1$ protein.

\section{PD-1-deficient did not affect cytotoxic and cytokine release, and induce apoptosis}

A549 cells were cocultured with different proportions of 


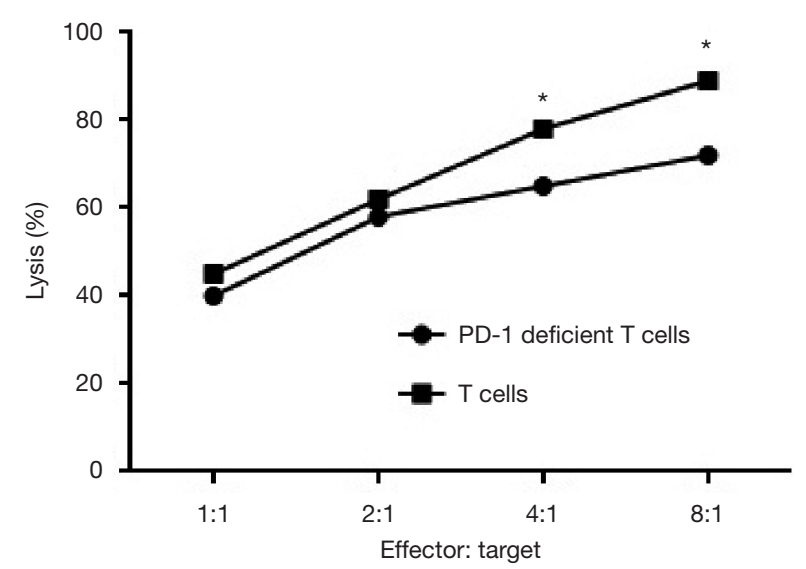

Figure 3 Anti-A549 cell effect of the ITSM mutation of PD-1 protein T cells. PD-1 deficient $T$ cells have stronger killing ability against A549 cells than T cells. ITSM, immune receptor tyrosine-based switch motif. *, significant difference between the two groups of data $(\mathrm{P}<0.05)$.

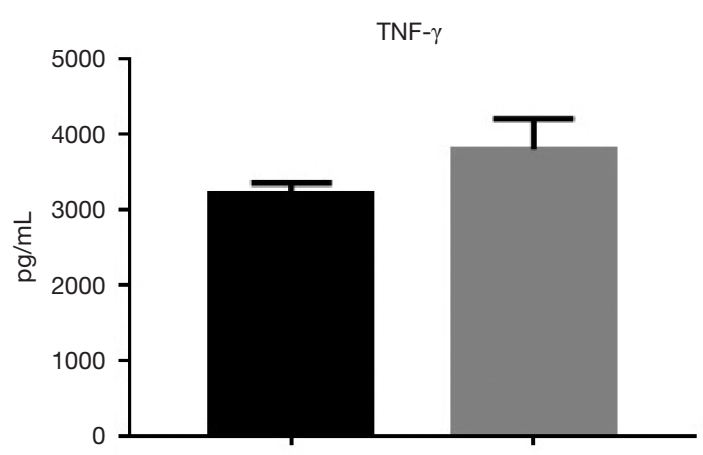

TNF- $\alpha$

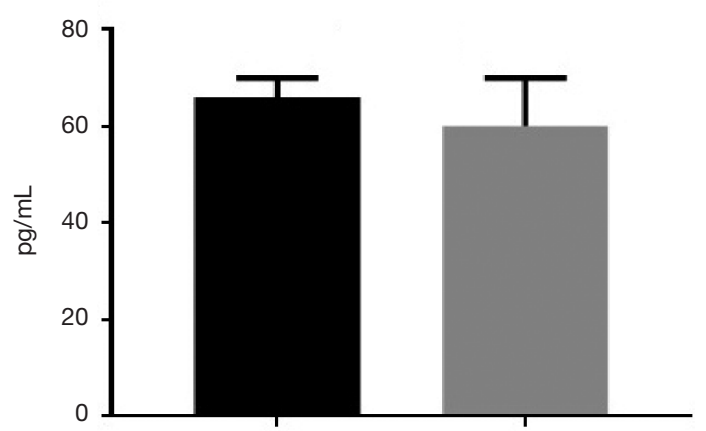

PD-1-deficient $\mathrm{T}$ and $\mathrm{T}$ cells. After $24 \mathrm{~h}$, the A549 cells were collected for counting. The PD-1-deficient $T$ and $T$ cells had a good killing effect on the target cells (A549), and the killing effect of the PD-1-deficient $T$ cells was better than that of the $T$ cells. These results proved that the mutation of PD-1 ITSM can enhance the antitumor effect of T cells in vitro (Figure 3).

No significant difference was observed in cytokine release between the PD-1-deficient $\mathrm{T}$ and $\mathrm{T}$ cells. The ITSM deficiency of PD-1 protein had no significant effect on the release of $\mathrm{T}$ cytokines (Figure 4). To explore whether apoptosis rate of ITSM mutation T cells was higher than wild type $\mathrm{T}$ cells, we quantified cell lysis apoptosis with annexin V/propidium staining. The results showed that ITSM mutation T cells showed no significant apoptosis compared with wild-type T cells (Figure 5).

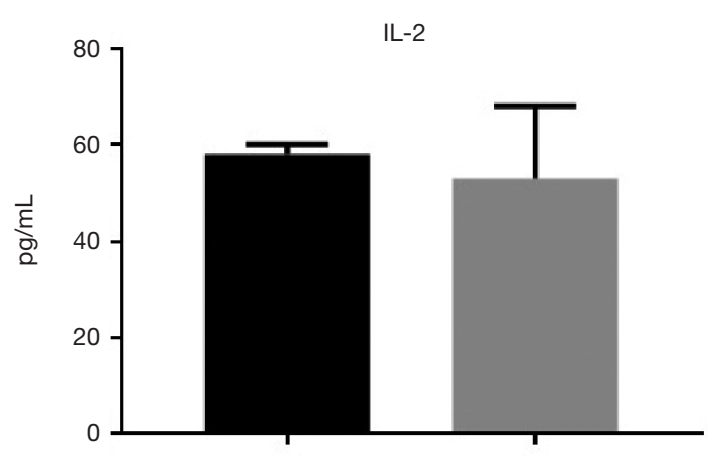

GM-CSF

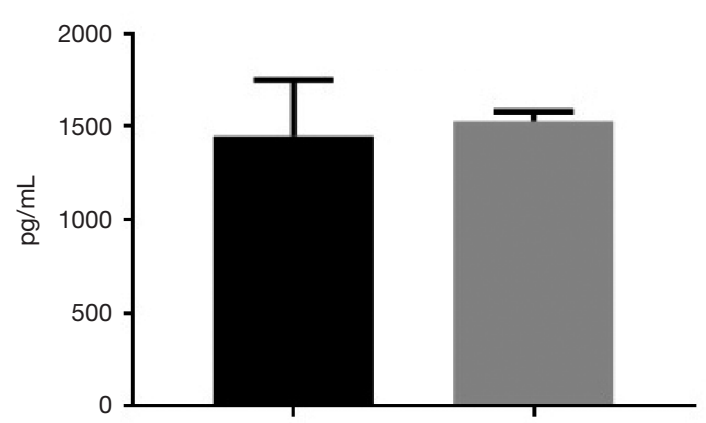

T cells

PD-1 deficient T cells

Figure 4 Comparison of cytokine secretion in PD-1-deficient $\mathrm{T}$ cells and $\mathrm{T}$ cells. There was no significant difference in the secretion function of various factors (TNF- $\gamma, \mathrm{IL}-2, \mathrm{TNF}-\alpha$ and GM-CSF) between PD-1 deficient T cells and T cells. This result indicated that PD-1 deficient T cells did not affect the function of T cells. TNF, tumor necrosis factor; IL-2, recombinant human interleukin-2; GM-CSF, granulocyte-macrophage colony-stimulating factor. 


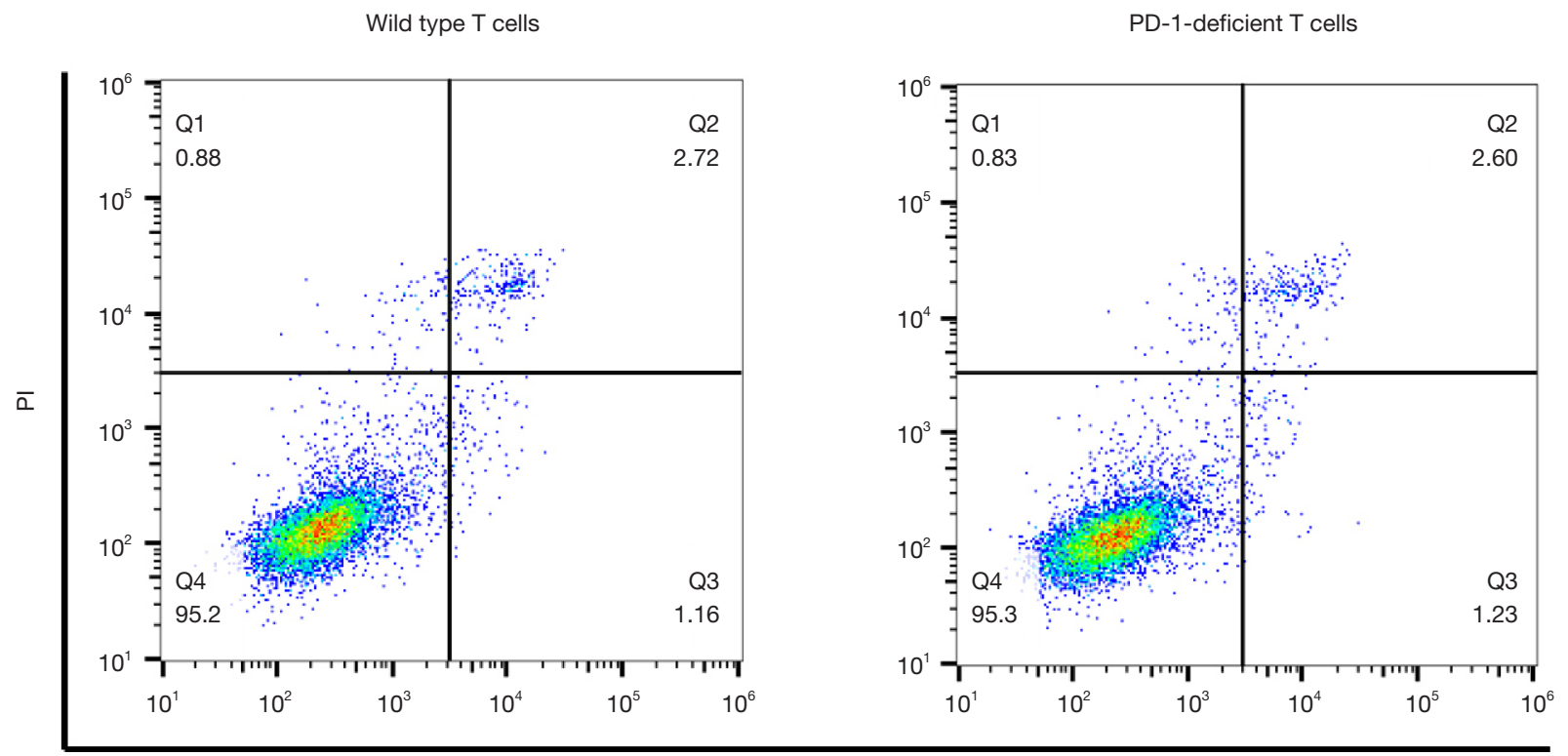

Annexin V-FITC

Figure 5 Apoptosis analysis of PD-1-deficient T cells. The effect of PD-1 ITSM motif mutation on T cells apoptosis was determined by flow cytometry.

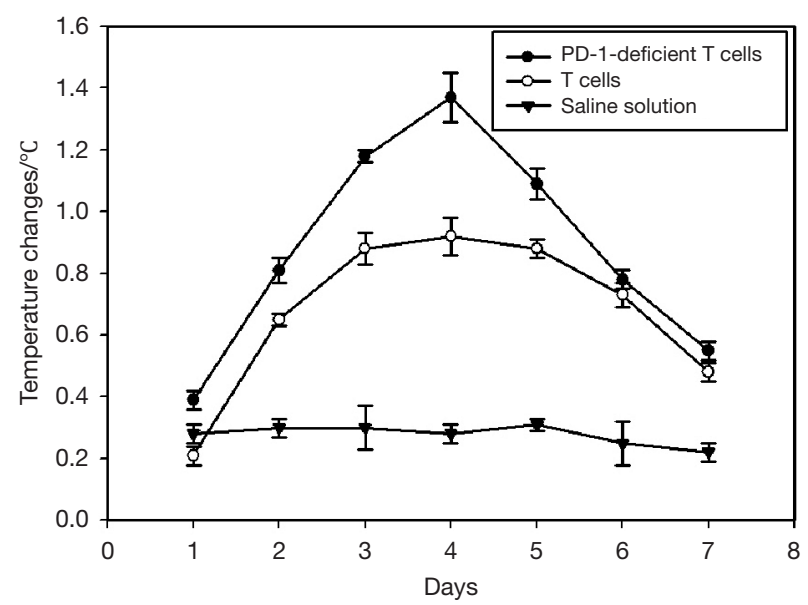

Figure 6 Change in body temperature of mouse. PD-1 deficient $\mathrm{T}$ cells, $\mathrm{T}$ cells and saline solution were infected into the mice by vein, respectively. PD-1 deficient $\mathrm{T}$ cells induce higher temperature changes than $\mathrm{T}$ cells and saline solution. This result indicated that PD-1 deficient $\mathrm{T}$ cells have more stronger killing ability against tumor of mouse.

\section{PD-1 deficiency enbanced tumor elimination efficacy of T cells in mouse models}

Before the first administration, the anus of mice was sterilized using electronic thermometer and alcohol. Then, the anus temperature of mice was measured simultaneously for seven days in each group, which indicated that the body temperature of the tumor-bearing mice can be changed after injecting the PD-1-deficient $\mathrm{T}$ or $\mathrm{T}$ cells. No mice died or showed adverse events in the course of the experiment, therefore, all 10 mice in each group were included in the analysis. The body temperature of tumor-bearing mice was the highest on the fourth day and then slowly decreased (Figure 6). The body weight of the experimental group injected with PD-1-deficient T cells had the smallest change (Figure 7). The Vernier caliper was used to measure the length and diameter of tumor in mice and draw the curve of tumor volume change (Figure 8). The tumor growth in mice treated with PD-1-deficient T cells was dramatically repressed compared with that in the control group. As such, the PD-1-deficient $T$ cells effectively inhibited the growth of human non-small cell lung cancer cells in xenografted mice. Interestingly, these results showed that the group treated with PD-1-deficient $T$ cells averaged $79.95 \%$ less than the control group.

\section{Discussion}

Many tumor therapies, such as antibody drug, genome editing, RNA interference, and other combinations, have been developed on the basis of the PD-1/PD-L1 signaling 


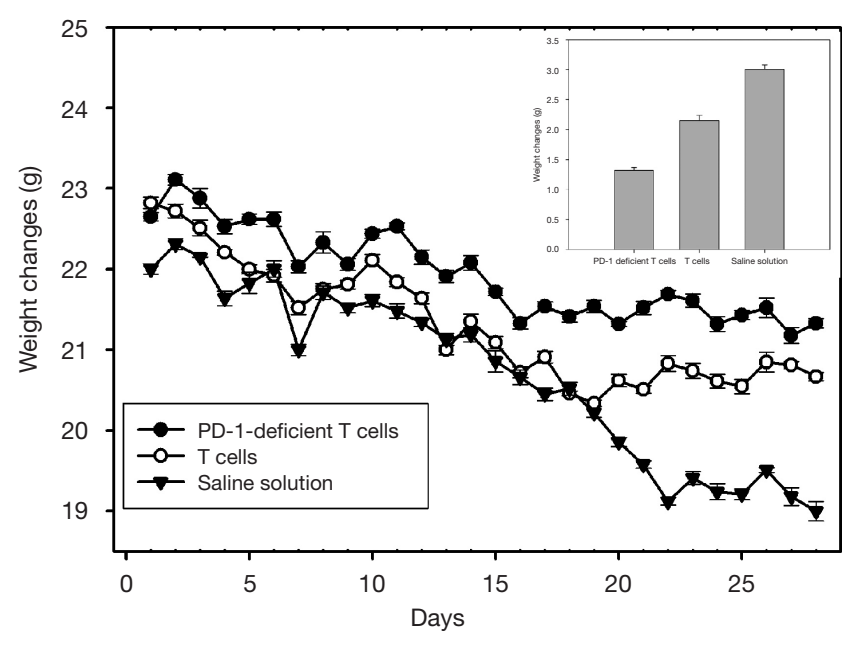

Figure 7 Change in body weight of mouse. The body weight change of saline solution and $\mathrm{T}$ cells group mouse were more significant than PD-1 deficient group mouse. The results indicated PD-1 deficient $T$ cells controlled the growth of tumor in the mouse.

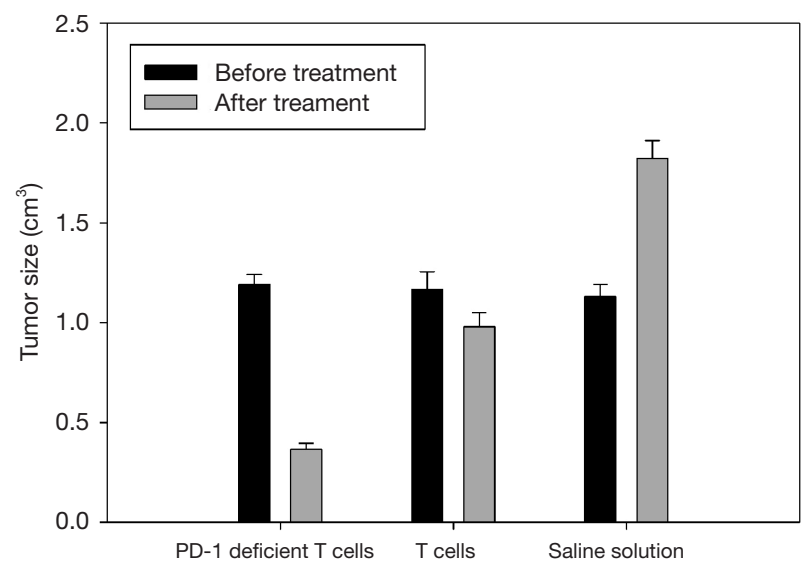

Figure 8 Change in tumor size in the xenograft mouse model (A549 cells). There was a significant reduction in tumor size of PD-1 deficient $\mathrm{T}$ cells mouse group than $\mathrm{T}$ cells mouse group. And the tumor size of saline solution mouse group grew as time prolongs.

pathway $(1,23)$. The PD-1 knockout or knockdown in T cells or CAR T cells can enhance T cell-mediated cytotoxicity, and less adverse reactions are observed $(10,24,25)$.

PD-1 is an important component of the B7/CD28 costimulatory superfamily. PD-1 is primarily expressed on the surface of activated $\mathrm{T}$ and $\mathrm{B}$ cells and consists of 268 amino acids. A membrane-penetrating glycoprotein is composed of acids, and its cytoplasmic region contains the immune receptor tyramine, acid ITIM, and ITSM (13). When PD-1 binds to ligands (PD-L1 and PD-L2), the phosphorylation of tyrosine residues in ITSM and the recruitment of phosphatase SHP-1 and SHP-2 can further dephosphorylate the downstream PI3K protein kinase; inhibit the activation of mTOR, ERK2, and Akt; and finally inhibit the T cell effect (13). The ITIM function is still not clear.

The genome base-editing technology has higher editing efficiency and less damage on the PD-1 protein structure and other functions than the universal CRIPSR/Cas9 technology (17-20). PD-1-edited T cells were manufactured ex vivo by co-transfection using electroporation of Cas9 and single gRNA plasmids and then were applied for 12 nonsmall cell lung cancer patients. The results showed that the median progression-free survival was 7.7 weeks (95\% confidence interval, 6.9-8.5 weeks) and median overall survival was 42.6 weeks (95\% confidence interval, $10.3-$ 74.9 weeks) (26).

In this study, the PD-1 ITSM was disrupted in cytotoxic $\mathrm{T}$ cells by using the $\mathrm{ABE}-\mathrm{xCas} 9$ system, and the antitumor effect of PD-1-deficient $\mathrm{T}$ cells on non-small cell lung cancer cells (A549 cell) was tested. Compared with the control $\mathrm{T}$ cells, the PD-1-deficient $\mathrm{T}$ cells more efficiently killed the tumor cells. The antitumor activity of PD-1-deficient $\mathrm{T}$ cells was verified in vivo by using a mouse xenograft model (A549). The treatment with PD1-deficient $T$ cells significantly inhibited the xenograft tumor growth and did not present adverse reactions to the host. The mechanistic study showed that the enhanced antitumor effect of ITSM on PD-1 protein was associated with increased apoptosis and augmented caspase activities in tumor cells. The secretion of cytokines (primarily TNF- $\alpha$ and IFN- $\gamma$ ) is one of the mechanisms by which T cells kill tumor cells. Our results showed that ITSM deficiency of PD-1 in T cells did not affect the cytokine secretion by $\mathrm{T}$ cells and did not induce apoptosis, which may be partly due to the increased antitumor activity of the PD-1-deficient $\mathrm{T}$ cells. This study expanded the arsenal of cancer therapy by showing the potential application of the ABE-xCas9 technology in targeting PD-1 immune checkpoint.

\section{Acknowledgments}

Funding: This work was supported by grants from the Zibo city innovation development key project (2018CX04A007); the Natural Scientific Foundation of Shandong Province, China (ZR2018MH038, ZR2019PC053); the National 
Natural Scientific Foundation of China (31701042); and the Zibo Platform for Gene Editing and Cell Application (2018ZBXC010, 2018ZBXC008).

\section{Footnote}

Reporting Checklist: The authors have completed the ARRIVE reporting checklist. Available at http://dx.doi. org/10.21037/tcr-20-2118

Data Sharing Statement: Available at http://dx.doi. org/10.21037/tcr-20-2118

Peer Review File: Available at http://dx.doi.org/10.21037/tcr20-2118

Conflicts of Interest: All authors have completed the ICMJE uniform disclosure form (available at http:// dx.doi.org/10.21037/tcr-20-2118). All authors have been issued with a patent for the preparation of PD-1 immunodeactivation checkpoint T-lymphocyte preparation.

Ethical Statement: The authors are accountable for all aspects of the work in ensuring that questions related to the accuracy or integrity of any part of the work are appropriately investigated and resolved. The experiments were conducted in accordance with the guidelines on the care and use of experimental animals issued by the Ministry of Health of the People's Republic of China and the guidelines on the care and use of animals in the IJSM ethical standard documents, and in accordance with the project license (No. 20200001) granted by the Animal Experiment Committee of Qilu Medical University.

Open Access Statement: This is an Open Access article distributed in accordance with the Creative Commons Attribution-NonCommercial-NoDerivs 4.0 International License (CC BY-NC-ND 4.0), which permits the noncommercial replication and distribution of the article with the strict proviso that no changes or edits are made and the original work is properly cited (including links to both the formal publication through the relevant DOI and the license). See: https://creativecommons.org/licenses/by-nc-nd/4.0/.

\section{References}

1. Chamoto K, Hatae R, Honjo T. Current issues and perspectives in PD-1 blockade cancer immunotherapy. Int
J Clin Oncol 2020;25:790-800.

2. Qin S, Xu L, Yi M, et al. Novel immune checkpoint targets: moving beyond PD-1 and CTLA-4. Mol Cancer 2019;18:155.

3. Zak KM, Grudnik P, Magiera K, et al. Structural Biology of the Immune Checkpoint Receptor PD-1 and Its Ligands PD-L1/PD-L2. Structure 2017;25:1163-74.

4. Su S, Hu B, Shao J, et al. CRISPR-Cas9 mediated efficient PD-1 disruption on human primary $\mathrm{T}$ cells from cancer patients. Sci Rep 2016;6:20070.

5. Zhao Z, Shi L, Zhang W, et al. CRISPR knock out of programmed cell death protein 1 enhances antitumor activity of cytotoxic T lymphocytes. Oncotarget 2018;9:5208-15.

6. Huang K, Sun B, Luo N, et al. Programmed Death Receptor 1 (PD1) Knockout and Human Telomerase Reverse Transcriptase (hTERT) Transduction Can Enhance Persistence and Antitumor Efficacy of CytokineInduced Killer Cells Against Hepatocellular Carcinoma. Med Sci Monit 2018;24:4573-82.

7. Su S, Zou Z, Chen F, et al. CRISPR-Cas9-mediated disruption of PD-1 on human T cells for adoptive cellular therapies of EBV positive gastric cancer. Oncoimmunology 2017;6:e1249558.

8. Rupp LJ, Schumann K, Roybal KT, et al. CRISPR/Cas9mediated PD-1 disruption enhances anti-tumor efficacy of human chimeric antigen receptor T cells. Sci Rep 2017;7:737.

9. Ren J, Zhang X, Liu X, et al. A versatile system for rapid multiplex genome-edited CAR T cell generation. Oncotarget 2017;8:17002-11.

10. Stadtmauer EA, Cohen AD, Weber K, et al. First-inHuman Assessment of Feasibility and Safety of Multiplexed Genetic Engineering of Autologous T Cells Expressing NY-ESO -1 TCR and CRISPR/Cas9 Gene Edited to Eliminate Endogenous TCR and PD-1 (NYCE T cells) in Advanced Multiple Myeloma (MM) and Sarcoma. Blood 2019;134:49.

11. Bardhan K, Aksoylar HI, Bourgeois TL, et al. Phosphorylation of PD-1-Y248 is a marker of PD-1mediated inhibitory function in human T cells. Sci Rep 2019;9:17252.

12. Sheppard KA, Fitz LJ, Lee JM, et al. PD-1 inhibits T-cell receptor induced phosphorylation of the ZAP70/CD3zeta signalosome and downstream signaling to PKCtheta. FEBS Lett 2004;574:37-41.

13. Daeron M, Jaeger S, Du Pasquier L, et al. Immunoreceptor tyrosine-based inhibition motifs: a quest in the past and 
future. Immunol Rev 2008;224:11-43.

14. Li B, Niu Y, Ji W, et al. Strategies for the CRISPR-Based Therapeutics. Trends Pharmacol Sci 2020;41:55-65.

15. Dever DP, Bak RO, Reinisch A, et al. CRISPR/Cas9 betaglobin gene targeting in human haematopoietic stem cells. Nature 2016;539:384-9.

16. Ureña-Bailén G, Lamsfus-Calle A, Daniel-Moreno A, et al. CRISPR/Cas9 technology: towards a new generation of improved CAR-T cells for anticancer therapies. Brief Funct Genomics 2020;19:191-200.

17. Lee HK, Smith HE, Liu C, et al. Cytosine base editor 4 but not adenine base editor generates off-target mutations in mouse embryos. Commun Biol 2020;3:19.

18. Wang X, Liu Z, Li G, et al. Efficient Gene Silencing by Adenine Base Editor-Mediated Start Codon Mutation. Mol Ther 2020;28:431-40.

19. McGrath E, Shin H, Zhang L, et al. Targeting specificity of APOBEC-based cytosine base editor in human iPSCs determined by whole genome sequencing. Nat Commun 2019;10:5353.

20. Liang P, Wen J, Huang J. Off-target effects of cytidine base editor and adenine base editor: What can we do? J

Cite this article as: Qi T, Fu J, Zhang W, Cui W, Xu X, Yue J, Wang Q, Tian X. Mutation of PD-1 immune receptor tyrosinebased switch motif (ITSM) enhances the antitumor activity of cytotoxic T cells. Transl Cancer Res 2020;9(11):6811-6819. doi: $10.21037 /$ tcr-20-2118
Genet Genomics 2019;46:509-12.

21. Gaudelli NM, Komor AC, Rees HA, et al. Programmable base editing of $A^{*} T$ to $G^{*} \mathrm{C}$ in genomic DNA without DNA cleavage. Nature 2017;551:464-71.

22. Komor AC, Kim YB, Packer MS, et al. Programmable editing of a target base in genomic DNA without doublestranded DNA cleavage. Nature 2016;533:420-4.

23. Borkner L, Kaiser A, van de Kasteele W, et al. RNA interference targeting programmed death receptor-1 improves immune functions of tumor-specific $T$ cells. Cancer Immunol Immunother 2010;59:1173-83.

24. Hu W, Zi Z, Jin Y, et al. CRISPR/Cas9-mediated PD-1 disruption enhances human mesothelin-targeted CAR T cell effector functions. Cancer Immunol Immunother 2019;68:365-77.

25. Choi BD, Yu X, Castano AP, et al. CRISPR-Cas9 disruption of PD-1 enhances activity of universal EGFRvIII CAR T cells in a preclinical model of human glioblastoma. J Immunother Cancer 2019;7:304.

26. Lu Y, Xue J, Deng T, et al. Safety and feasibility of CRISPR-edited $T$ cells in patients with refractory nonsmall-cell lung cancer. Nat Med 2020;26:732-40. 